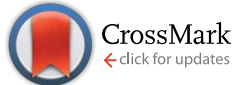

Cite this: Chem. Sci., 2015, 6, 259

Received 6th August 2014 Accepted 12th September 2014

DOI: $10.1039 / \mathrm{c} 4 \mathrm{sc} 02377 \mathrm{c}$

www.rsc.org/chemicalscience

\title{
An aqueous molecular tube with polyaromatic frameworks capable of binding fluorescent dyes $\uparrow$
}

\author{
Keita Hagiwara, Munetaka Akita and Michito Yoshizawa* \\ An aqueous molecular tube composed of polyaromatic frameworks with peripheral hydrophilic groups was \\ prepared. The new tube has a well-defined hydrophobic cavity with a diameter of $\sim 1 \mathrm{~nm}$ and quantitatively \\ binds two molecules of fluorescent coumarin dyes in aqueous solutions. The bound coumarin dimers in a \\ stacked fashion exhibit unusual excimer-like emissions in the confined space through efficient host-guest \\ energy transfer.
}

\section{Introduction}

Molecular rings and tubes composed of entirely aromatic frameworks, as contrasted with well-studied aliphatic ones (e.g., cyclodextrins and cucurbiturils), have recently attracted greater attention from the viewpoint of both synthetic and physical chemistries due to their relevance to the partial structures of single-walled carbon nanotubes. ${ }^{\mathbf{1 , 2}}$ Such aromatic macrocycles have been prepared by linking multiple benzene, naphthalene, pyrene, or chrysene rings ${ }^{3,4}$ through transition metal-mediated coupling reactions and they exhibit intriguing fluorescence properties based on their aromatic frameworks. However, their host-guest interactions have been limited to large spherical molecules, i.e. fullerenes, ${ }^{5}$ probably due to their large hydrophobic nature. Moreover, the supramolecular host-guest photophenomena of the aromatic macrocycles, as well as previous aliphatic tubes, have been relatively unexplored..$^{6,7} \mathrm{We}$ anticipated that the development of water-soluble molecular tubes with polyaromatic frameworks would lead to establishment of new photofunctional molecular flasks, providing wide-ranging host capabilities and unique host-guest photophysical properties (Fig. 1a). Here we present the preparation of a new aqueous molecular tube $\mathbf{1}$ with polyaromatic anthracene panels and peripheral hydrophilic sulfonate groups $\left(\mathrm{R}=-\mathrm{OCH}_{2} \mathrm{CH}_{2} \mathrm{CH}_{2}\right.$ $\mathrm{SO}_{3}{ }^{-} \cdot \mathrm{Na}^{+}$; Fig. 1b). The tube quantitatively binds two molecules of fluorescent coumarin dyes in aqueous solutions at room temperature. We emphasize that the bound coumarin dimers exhibit unusual excimer-like emissions in the confined tubular cavity through efficient energy transfer from the host framework to the guest dimers.

Recently, we have reported molecular tube $\mathbf{1}^{\prime}\left(\mathrm{R}=-\mathrm{OCH}_{3}\right.$ and $-\mathrm{OCH}_{2} \mathrm{CH}_{2} \mathrm{OCH}_{3}$ ) composed of four anthracene panels ${ }^{\mathbf{8}, 9}$

Chemical Resources Laboratory, Tokyo Institute of Technology, 4259 Nagatsuta, Midori-ku, Yokohama 226-8503, Japan.E-mail: yoshizawa.m.ac@m.titech.ac.jp

$\uparrow$ Electronic supplementary information (ESI) available: Experimental procedures and physical data. See DOI: $10.1039 / \mathrm{c} 4 \mathrm{sc} 02377 \mathrm{c}$ linked by phenylene and biphenylene spacers at the meta positions (Fig. 1b). ${ }^{10}$ The tubular structure has a well-defined 1 nm-sized cavity surrounded by hydrophobic anthracene frameworks (Fig. 1c), however its synthetic protocol demands a multistep preparation (15 steps; Scheme S1†) including a crosscoupling reaction between triflate-capped and boronate-capped
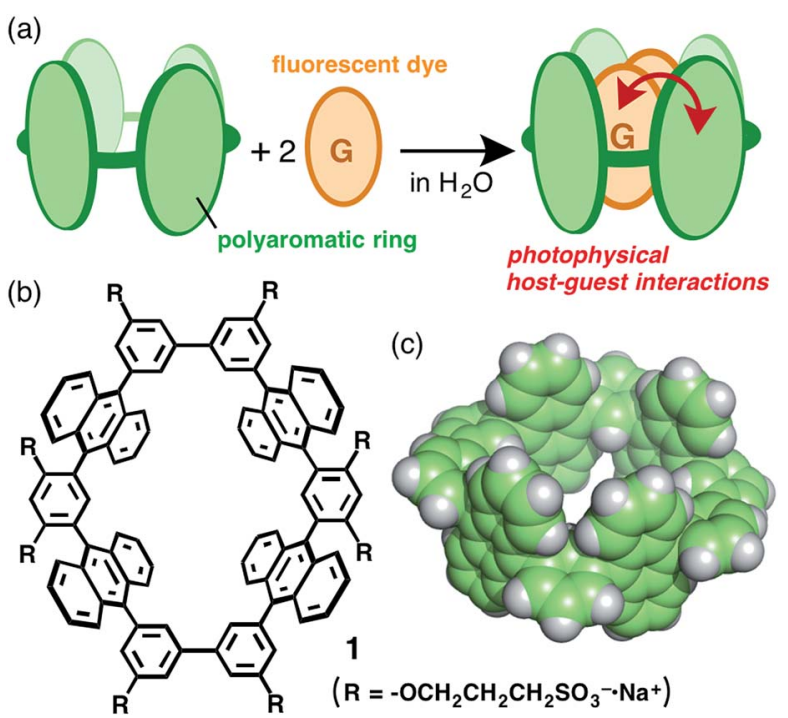

(d)

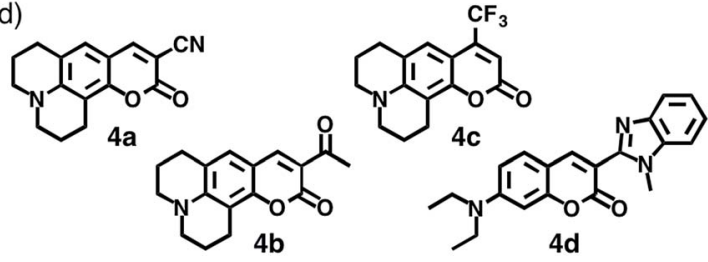

Fig. 1 (a) Schematic representation of the encapsulation of guest molecules by an aqueous molecular tube with polyaromatic frameworks. (b) Aqueous molecular tube 1 and (c) the polyaromatic framework $(\mathrm{R}=-\mathrm{H})$. (d) Coumarin dyes $4 \mathrm{a}-\mathrm{d}$ employed as guest molecules. 
half-tubes, which limits its further exploration. Besides, no host-guest interactions have been observed for the hydrophobic tube owing to its solubility in only organic solvents (e.g., $\mathrm{CHCl}_{3}$ and acetone).

\section{Results and discussion}

\section{Synthesis of an aqueous molecular tube}

Water-soluble molecular tube $\mathbf{1}$ was obtained from anthracene dimer 3a by the following straightforward synthetic route, which is 7-steps shorter than the previous route and includes bromo-capped half-tube $\mathbf{2 a}$ as a key intermediate (Fig. 2 and ESI $\dagger$ ). After the iodination of 3a, the Suzuki-Miyaura coupling of $\mathbf{3 b}(\mathrm{X}=-\mathrm{I})$ with 3-bromo-5-methoxyphenyl boronate afforded $2 \mathrm{a}$ in $54 \%$ yield. After the methoxy groups on $\mathbf{2 a}$ were converted into 2-methoxymethoxy groups, a homo-coupling reaction of the methoxymethoxy-protected $2 \mathbf{b}$ using a $\mathrm{Ni}(\operatorname{cod})_{2} /$ bipyridine catalyst $^{\mathbf{1 1}}$ gave rise to tube $\mathbf{1}^{\prime \prime}$ in a moderate yield (30\%). Conversion of the peripheral substituents on $\mathbf{1}^{\prime \prime}$ to hydrophilic sulfonate groups by sequential deprotection and etherification gave rise to aqueous tube 1 in $50 \%$ yield ( 2 steps).
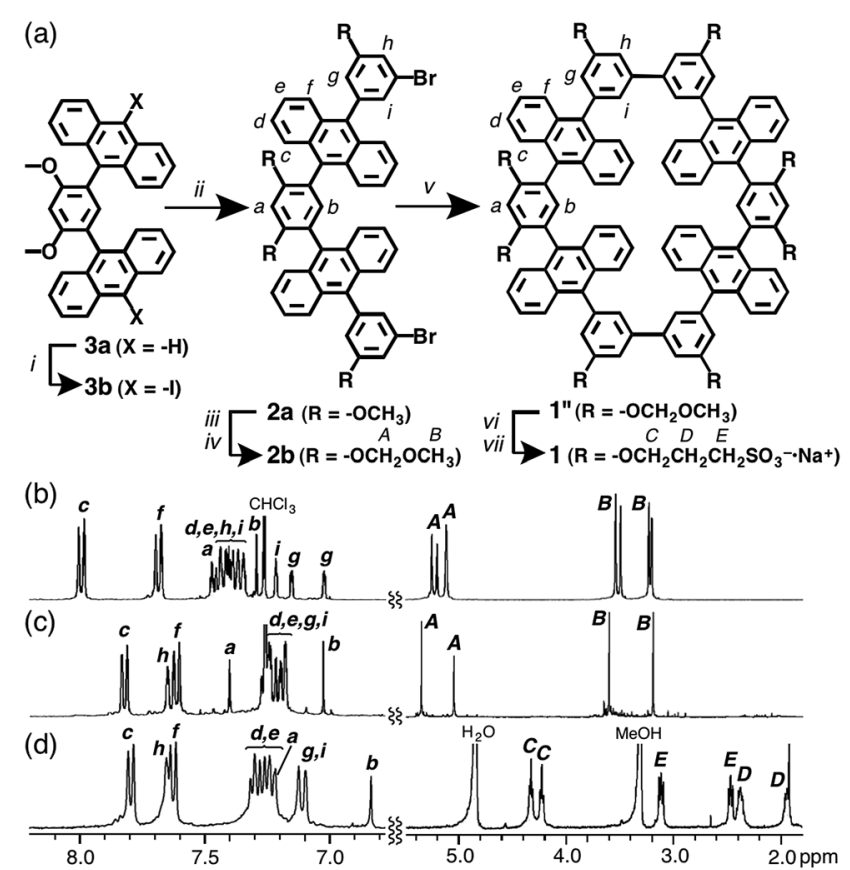

(e) $\left[1-8 \mathrm{Na}^{+}\right]^{8-}$

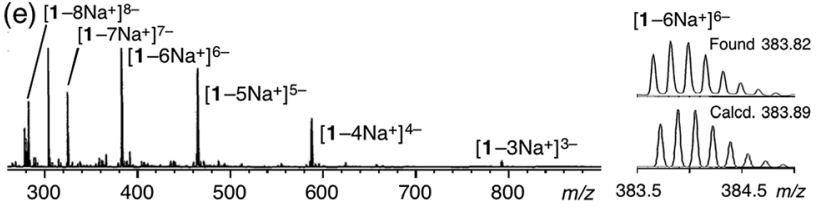

Fig. 2 (a) Preparation of aqueous molecular tube 1. Reagents and catalysts: (i) 1,3-diiodo-5,5-dimethylhydantoin; (ii) 3-bromo-5methoxyphenylboronic acid pinacol ester, $\mathrm{Pd}\left(\mathrm{PPh}_{3}\right)_{4}, \mathrm{Na}_{2} \mathrm{CO}_{3}$; (iii) $\mathrm{BBr}_{3}$; (iv) chloromethyl methyl ether, $\mathrm{Cs}_{2} \mathrm{CO}_{3} ;$ (v) $\mathrm{Ni}(\mathrm{cod})_{2}, 2,2^{\prime}$ bipyridyl; (vi) $\mathrm{HCl}_{\text {aq.i }}$ ( vii) 1,3-propanesultone, $\mathrm{NaH} .{ }^{1} \mathrm{H}$ NMR spectra (400 MHz, r.t.) of (b) bromo-capped half-tube $2 \mathrm{~b}$ and (c) hydrophobic tube $1^{\prime \prime}$ in $\mathrm{CDCl}_{3}$, and (d) aqueous tube 1 in $\mathrm{CD}_{3} \mathrm{OD}$. (e) ESI-TOF MS spectrum of aqueous tube 1 in $\mathrm{CH}_{3} \mathrm{OH}$ with an expansion and simulation of the $\left[1-6 \mathrm{Na}^{+}\right]^{6-}$ signal.
The structures of molecular tubes 1 and half-tubes 2 were fully confirmed by NMR spectroscopy and mass spectrometry. $\dagger$ The ${ }^{1} \mathrm{H}$ NMR spectrum of tube precursor $2 \mathbf{b}$ (Fig. 2b) showed the corresponding aromatic $\mathrm{H}_{\mathrm{a}-\mathrm{i}}$ and aliphatic $\mathrm{H}_{\mathrm{A}, \mathrm{B}}$ signals as assigned by COSY and NOESY analyses. Characteristically, two sets of proton signals derived from the functionalized phenyl moieties $\left(\mathrm{H}_{\mathrm{g}, \mathrm{i}}\right.$ and $\left.\mathrm{H}_{\mathrm{A}, \mathrm{B}}\right)$ were observed in a $1: 1$ ratio. ${ }^{12}$ On the other hand, the ${ }^{1} \mathrm{H}$ NMR analysis of $\mathbf{1}^{\prime \prime}$ showed a simple spectrum displaying nine aromatic and four aliphatic signals (Fig. 2c). This spectral change is reasonable upon the formation of tubular compound $\mathbf{1}^{\prime \prime}$ with $D_{2 \mathrm{~h}}$ symmetry. Matrix-assisted laser desorption ionization time-of-flight mass spectrometry (MALDI-TOF MS) revealed the formation of $\mathbf{1}^{\prime \prime}$ with a prominent peak at $m / z=1641.44$. After isolation of aqueous tube 1 , the ${ }^{1} \mathrm{H}$ NMR spectrum in $\mathrm{CD}_{3} \mathrm{OD}$ exhibited new aliphatic signals attributed to the decorated sulfonate groups $\left(\mathrm{H}_{\mathrm{C}-\mathrm{E}}\right)$ in the range 4.4-1.8 ppm (Fig. 2d). Electrospray ionization (ESI)-TOF MS spectrum of 1 showed a series of intense peaks at e.g., 791.0 $\left[1-3 \mathrm{Na}^{+}\right]^{3-}, 587.2\left[1-4 \mathrm{Na}^{+}\right]^{4-}$, and $465.2\left[1-5 \mathrm{Na}^{+}\right]^{5-}$ (Fig. 2e), which unambiguously elucidated full modification of the tube by the hydrophilic groups.

Obtained sulfonate-coated tube $\mathbf{1}$ is highly soluble in water ( $>100 \mathrm{mM}$ ) but tubes $\mathbf{1}^{\prime}$ and $\mathbf{1}^{\prime \prime}$ are effectively insoluble in water. In the ${ }^{1} \mathrm{H}$ NMR spectrum of $\mathbf{1}$ in $\mathrm{D}_{2} \mathrm{O}$, the proton signals were significantly broadened (Fig. S20†), most probably originating from slow intramolecular motion on the NMR timescale. Dynamic light scattering (DLS) analysis of aqueous solutions of 1 showed small particles with an average diameter of $1.4 \mathrm{~nm}$ (Fig. S21a $\dagger$ ), which indicates no aggregation of 1 in water. An optimized structure of 1 shows that its external and core diameters are approximately 1.9 and $1.0 \mathrm{~nm}$, respectively (Fig. 3a).

The UV-vis spectrum of tube $\mathbf{1}$ in $\mathrm{H}_{2} \mathrm{O}$ exhibited the typical $\pi-\pi^{*}$ transition bands of the anthracene moieties (Fig. 3b). The

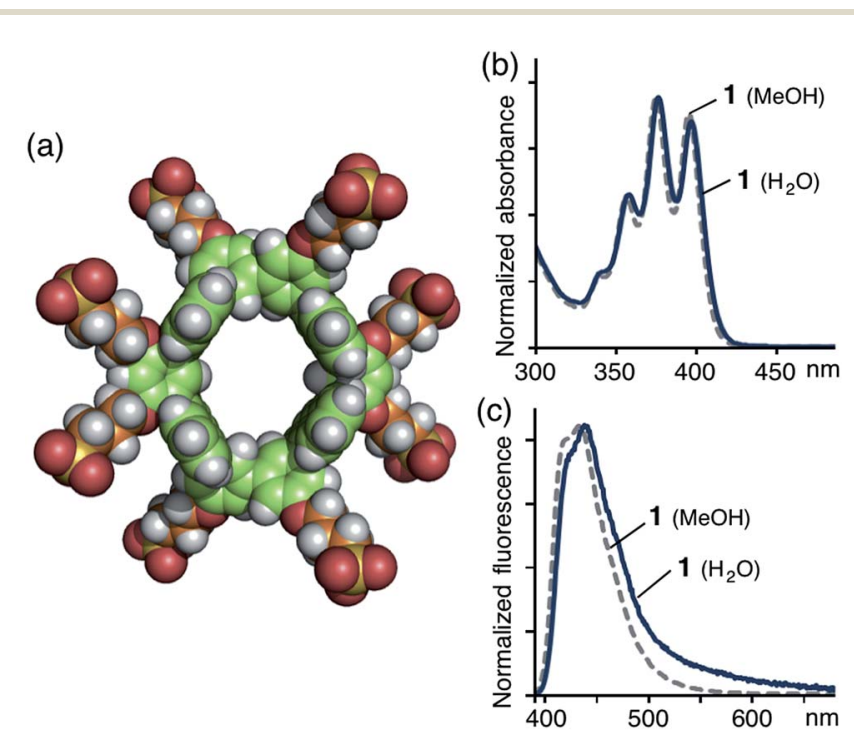

Fig. 3 (a) An optimized structure of aqueous tube 1 without counterions or solvents by PM3 calculation. (b) UV-vis spectra (10 $\mu \mathrm{M}$, r.t.) and (c) fluorescence spectra ( $\lambda_{\mathrm{ex}}=378 \mathrm{~nm}, 10 \mu \mathrm{M}$, r.t.) of 1 in $\mathrm{H}_{2} \mathrm{O}$ (solid line) and $\mathrm{CH}_{3} \mathrm{OH}$ (dashed line). 
emission spectra of 1 in $\mathrm{H}_{2} \mathrm{O}$ and $\mathrm{CH}_{3} \mathrm{OH}$ displayed a broad band in the range $390-550 \mathrm{~nm}\left(\lambda_{\max }=\sim 440 \mathrm{~nm}\right.$; Fig. $\left.3 \mathrm{c}\right)$ with good absolute quantum yields $\left(\Phi_{\mathrm{F}}=30\right.$ and $59 \%$, respectively).$\dagger$ Thus, the obtained polyaromatic nanotube providing both good water-solubility and fluorescence properties would be expected to act as a photofunctional molecular host in aqueous media.

\section{Encapsulation of coumarin dyes}

The open-ended and $1 \mathrm{~nm}$-length cylindrical cavity of tube $\mathbf{1}$ facilitated the quantitative encapsulation of two molecules of coumarin 337 (4a) and 334 (4b), well-known hydrophobic fluorescent dyes, in aqueous solutions through hydrophobic and aromatic-aromatic interactions (Fig. 1a and d). When a slight excess of 3-cyano-substituted $\mathbf{4 a}$ was suspended in an aqueous solution $(0.2 \mathrm{mM})$ of tube 1 at room temperature for $1 \mathrm{~h}$, the color of the solution obviously changed from colorless to yellow due to the formation of a $1: 2$ host-guest complex, $1 \supset(\mathbf{4 a})_{2}$, as revealed by UV-vis and MS analyses. ${ }^{13}$ The UV-vis absorption spectrum of the product showed a new prominent band at $\lambda_{\max }=446 \mathrm{~nm}$ corresponding to the bound coumarin guests (Fig. 4a). The band is considerably narrower than that of free $\mathbf{4 a}$ in $\mathrm{CH}_{3} \mathrm{OH}$ (Fig. $\mathrm{S} 24 \dagger$ ). It is worthy of note that another new absorption band was observed as a shoulder at around $480 \mathrm{~nm}$, which can presumably be attributed to a $\pi$-stacked coumarin dimer. In combination with ESI-TOF MS analysis, ${ }^{\mathbf{1 4}}$ a UV-vis spectral titration study clearly illustrated the quantitative formation of the $1: 2$ host-guest structure (Fig. 4b). $\uparrow$ Similarly, two molecules of 3-acetyl-substituted coumarin $\mathbf{4 b}$ were quantitatively bound in the cavity of tube 1 to produce $\mathbf{1} \supset(\mathbf{4 b})_{2}$, which showed an absorption band for the guests at $\lambda_{\max }=455$ $\mathrm{nm}$ (Fig. 4a). Notably, the absorption bands of $\mathbf{4 a}$ and $\mathbf{4 b}$ overlap considerably with the emission band of $\mathbf{1}$ (Fig. 3c). An optimized
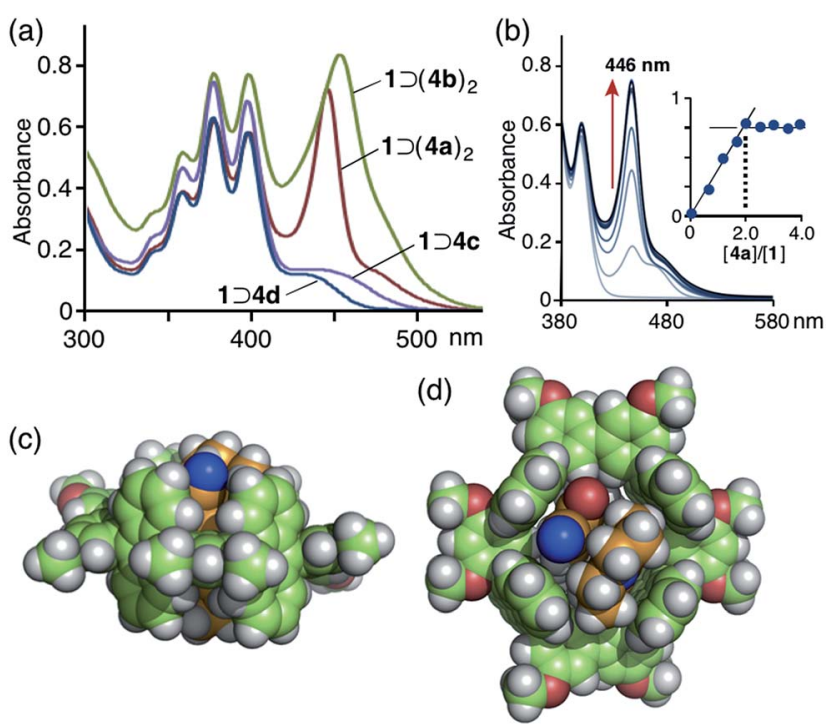

Fig. 4 (a) UV-vis spectra $\left(0.2 \mathrm{mM}, \mathrm{H}_{2} \mathrm{O}\right.$, r.t.) of $1 \supset(4 \mathrm{a})_{2}, 1 \supset(4 \mathrm{~b})_{2}, 1 \supset 4 \mathrm{c}$, and $1 \supset 4 \mathrm{~d}$. (b) Titration UV-vis spectra $\left(0.2 \mathrm{mM}, \mathrm{H}_{2} \mathrm{O}\right.$, r.t.) and the plot $\left(\lambda_{\text {abs }}=446 \mathrm{~nm}\right)$ of 1 with the addition of $4 \mathrm{a}([4 \mathrm{a}] /[1]=0.5,1.0,1.5,2.0$, $2.5,3.0,3.5,4.0)$. Optimized structure of $1 \supset(4 a)_{2}\left(R=-\mathrm{OCH}_{3}\right)$ : (c) side and (d) top views. structure of $1 \supset(\mathbf{4 a})_{2}\left(\mathrm{R}=-\mathrm{OCH}_{3}\right)$ obtained by force-field calculations ${ }^{\mathbf{1 3 , 1 5}}$ indicated that the two coumarin guests adopt a $\pi$-stacked anti-head-to-tail conformation with an interplanar distance of $3.5 \AA$ in the cavity (Fig. 4c and d). The stacked dimer fully occupies the nanospace and is in tight contact with the four anthracene panels of the tube.

Interestingly, in the case of 4-trifluoromethyl-substituted coumarin 153 (4c) and 3-( $N$-methyl)benzimidazolyl-substituted coumarin 30 (4d), tube 1 recognized the substituents on the coumarin dyes and yielded $1: 1$ host-guest complexes. ${ }^{10} \mathrm{UV}$-vis and ESI-TOF MS studies revealed the formation of $1 \supset \mathbf{4 c}$ and $1 \supset$ 4d (in 40 and 30\% yields, respectively) under conditions similar to those used for $\mathbf{1} \supset(\mathbf{4 a})_{2}$. The UV-vis spectra showed new absorption bands resulting from bound coumarins $4 \mathbf{c}$ and 4d at $\lambda_{\max }=437$ and $430 \mathrm{~nm}$, respectively (Fig. 4a). Force-field calculation studies suggested that the 4-trifluoromethyl and $N$-methylbenzimidazolyl groups on the coumarin dyes prevent the formation of $1: 2$ host-guest structures and efficient hostguest stacking interactions due to steric hindrance (Fig. S34 and $\mathrm{S} 38 \dagger$ ). The competitive binding experiment for tube 1 revealed the preferential binding series of $4 \mathrm{a} \approx 4 \mathrm{~b} \gg \mathbf{4 c} \approx \mathbf{4 d}$ for the coumarin guests. $\dagger$

\section{Unique host-guest photophysical properties}

The aqueous solutions of $1: 2$ host-guest complexes $1 \supset(\mathbf{4 a})_{2}$ and $1 \supset(\mathbf{4 b})_{2}$ exhibited unusual emissions from the stacked coumarin dimers upon irradiation of the host framework at $378 \mathrm{~nm}$ at room temperature. The fluorescence spectrum of $1 \supset(\mathbf{4 a})_{2}$ contained a new broad band at around $590 \mathrm{~nm}$, assignable to an excimer-like emission from $(\mathbf{4 a})_{2}$ within $\mathbf{1},{ }^{\mathbf{1 6}}$ whereas emission from the anthracene moieties of $1(\sim 440 \mathrm{~nm})$ and the monomeric coumarin guest $(\sim 510 \mathrm{~nm})$ were markedly weaker (Fig. 5a) due to efficient fluorescence resonance energy transfer $(\text { FRET })^{\mathbf{1 7}}$ from host $\mathbf{1}$ to guest $(\mathbf{4 a})_{2}$. Similarly, $\mathbf{1} \supset(\mathbf{4 b})_{2}$ showed an excimer-like emission band at around $600 \mathrm{~nm}$ in the

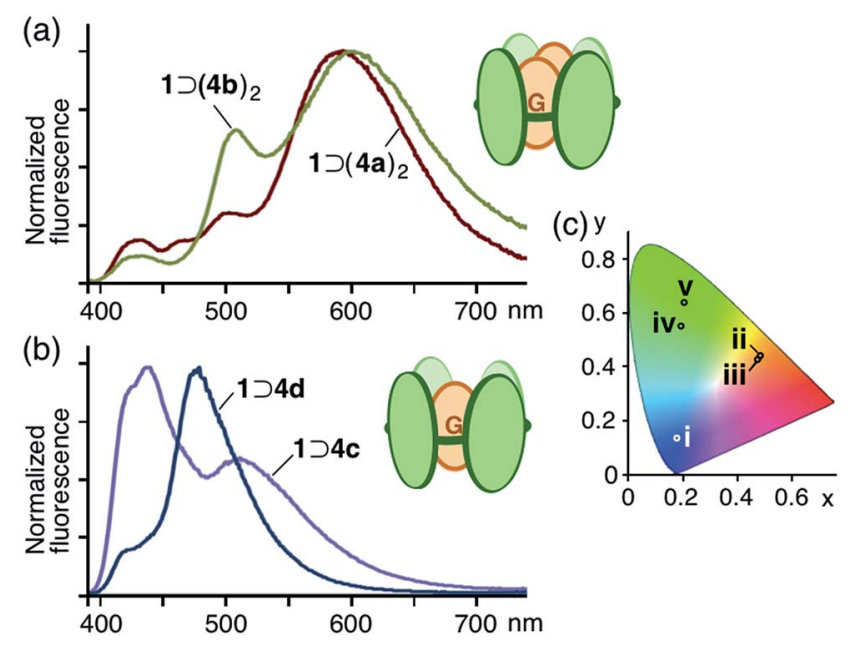

Fig. 5 Fluorescence spectra $\left(\lambda_{\mathrm{ex}}=378 \mathrm{~nm}, \mathrm{H}_{2} \mathrm{O}\right.$, r.t.) of (a) the $1: 2$ host-guest complexes and (b) the $1: 1$ host-guest complexes. (c) CIE chromaticity diagram of the fluorescence color of (i) 1 , (ii) $1 \supset(4 a)_{2}$, (iii) $1 \supset(4 \mathrm{~b})_{2}$ in $\mathrm{H}_{2} \mathrm{O}$ and (iv) $4 \mathrm{a}$, (v) $4 \mathrm{~b}$ in $\mathrm{CH}_{3} \mathrm{OH}$. 
fluorescence spectrum, accompanying a weak emission band $(\sim 500 \mathrm{~nm})$ for the monomeric coumarin guest. In sharp contrast, the aqueous solution of a mixture of $1 \supset 4 \mathbf{c}$ and 1 displayed only host and guest emissions ( $\sim 40$ and $\sim 510 \mathrm{~nm}$, respectively) and a mixture of $\mathbf{1} \supset \mathbf{4 d}$ and $\mathbf{1}$ displayed a prominent guest emission at $\lambda_{\max }=477 \mathrm{~nm}$ through FRET upon irradiation at $378 \mathrm{~nm}$ (Fig. 5b). The CIE chromaticity diagram for $\mathbf{1} \supset(\mathbf{4 a})_{2}$ and $\mathbf{1} \supset(\mathbf{4 b})_{2}$ quantified the emission colors as orange $((0.48,0.44)$ and $(0.48,0.43)$, respectively), unlike that for 1, 4a, and $4 \mathbf{b}$ (Fig. 5c).

The water-solubility of tube $\mathbf{1}$ is essential to both the strong binding of the hydrophobic fluorescent dyes and the unique emissions of the bound dyes through efficient host-guest and guest-guest interactions. When $\mathbf{1} \supset(\mathbf{4 a})_{2}$ was dissolved in $\mathrm{MeOH}$, the host-guest complex dissociated immediately. In the fluorescence spectrum, only emission bands from free $\mathbf{1}$ and $\mathbf{4 a}$ were observed upon irradiation at $378 \mathrm{~nm}$ (Fig. S24†).

\section{Conclusions}

In conclusion, we have succeeded in the preparation of a molecular tube with polyaromatic panels and peripheral hydrophilic groups. The new aqueous tube quantitatively binds two molecules of fluorescent coumarin dyes in its well-defined nanospace through hydrophobic and aromatic-aromatic interactions in water. Notably and most importantly, the bound fluorescent dyes in a stacked fashion exhibited excimer-like emission through efficient host-guest intermolecular interactions. This study demonstrates that photoactive and watersoluble polyaromatic tubes have potential for exploring novel host-guest supramolecular structures and subsequent unique photophysical properties.

\section{Acknowledgements}

This work was supported by the Japan Society for the Promotion of Science (JSPS) through a "Funding Program for Next-Generation World-Leading Researchers (NEXT Program)" and by the Japanese Ministry of Education, Culture, Sports, Science and Technology (MEXT) through Grants-in-Aid for Scientific Research on Innovative Areas "Soft Molecular Systems". K.H. thanks the JSPS for a Research Fellowship for Young Scientists.

\section{Notes and references}

1 (a) S. Iijima, Nature, 1991, 354, 56-58; (b) Carbon Nanotubes: Synthesis, Structure, Properties and Applications, ed. M. Dresselhaus, G. Dresselhaus and P. Avouris, Springer, Berlin, 2001.

2 (a) T. Kawase and H. Kurata, Chem. Rev., 2006, 106, 52505273; (b) K. Tahara and Y. Tobe, Chem. Rev., 2006, 106, 5274-5290; (c) M. Iyoda, J. Yamakawa and M. J. Rahman, Angew. Chem., Int. Ed., 2011, 50, 10522-10553; (d) H. Omachi, Y. Segawa and K. Itami, Acc. Chem. Res., 2012, 45, 1378-1389.

3 (a) C. Grave and A. D. Schlüter, Eur. J. Org. Chem., 2002, 30753098; (b) R. Jasti, J. Bhattacharjee, J. B. Neaton and
C. R. Bertozzi, J. Am. Chem. Soc., 2008, 130, 17646-17647; (c) H. Takaba, H. Omachi, Y. Yamamoto, J. Bouffard and K. Itami, Angew. Chem., Int. Ed., 2009, 48, 6112-6116; (d) S. Yamago, Y. Watanabe and T. Iwamoto, Angew. Chem., Int. Ed., 2010, 49, 757-759; (e) T. Iwamoto, Y. Watanabe, Y. Sakamoto, T. Suzuki and S. Yamago, J. Am. Chem. Soc., 2011, 133, 8354-8361; (f) T. J. Sisto, M. R. Golder, E. S. Hirst and R. Jasti, J. Am. Chem. Soc., 2011, 133, 1580015802.

4 (a) A. Yagi, Y. Segawa and K. Itami, J. Am. Chem. Soc., 2012, 134, 2962-2965; (b) S. Hitosugi, W. Nakanishi, T. Yamasaki and H. Isobe, Nat. Commun., 2011, 2, 492; (c) T. Iwamoto, E. Kayahara, N. Yasuda, T. Suzuki and S. Yamago, Angew. Chem., Int. Ed., 2014, 53, 6430-6434.

5 (a) T. Iwamoto, Y. Watanabe, T. Sadahiro, T. Haino and S. Yamago, Angew. Chem., Int. Ed., 2011, 50, 8342-8344; (b) H. Isobe, S. Hitosugi, T. Yamasaki and R. Iizuka, Chem. Sci., 2013, 4, 1293-1297; (c) S. Hitosugi, R. Iizuka, T. Yamasaki, R. Zhang, Y. Murata and H. Isobe, Org. Lett., 2013, 15, 3199-3201; (d) Y. Nakanishi, H. Omachi, S. Matsuura, Y. Miyata, R. Kitaura, Y. Segawa, K. Itami and H. Shinohara, Angew. Chem., Int. Ed., 2014, 53, 3102-3106.

6 (a) J. W. Steed and J. L. Atwood, Supramolecular Chemistry, Wiley, Chichester, U.K., 2nd edn, 2009; (b) F. Hof, S. L. Craig, C. Nuckolls and J. Rebek Jr, Angew. Chem., Int. Ed., 2002, 41, 1488-1508; (c) N. Sakai, J. Mareda and S. Matile, Acc. Chem. Res., 2008, 41, 1354-1365; (d) M. Yoshizawa, J. K. Klosterman and M. Fujita, Angew. Chem., Int. Ed., 2009, 48, 3418-3438; (e) H. Amouri, C. Desmarets and J. Moussa, Chem. Rev., 2012, 112, 20152041; (f) T. R. Cook, Y.-R. Zheng and P. J. Stang, Chem. Rev., 2013, 113, 734-777; (g) M. M. J. Smulders, I. A. Riddell, C. Browne and J. R. Nitschke, Chem. Soc. Rev., 2013, 42, 1728-1754; (h) G. Zhang and M. Mastalerz, Chem. Soc. Rev., 2014, 43, 1934-1947.

7 A recent water-soluble tube (without polyaromatic rings and fluorescent properties): Z. Li, J. Yang, G. Yu, J. He, Z. Abliz and F. Huang, Chem. Commun., 2014, 50, 2841-2843.

8 Multiple anthracene assemblies: (a) N. Kishi, Z. Li, K. Yoza, M. Akita and M. Yoshizawa, J. Am. Chem. Soc., 2011, 133, 11438-11441; (b) Z. Li, N. Kishi, K. Yoza, M. Akita and M. Yoshizawa, Chem.-Eur. J., 2012, 18, 8358-8365; (c) K. Kondo, A. Suzuki, M. Akita and M. Yoshizawa, Angew. Chem., Int. Ed., 2013, 52, 2308-2312; (d) A. Suzuki, K. Kondo, M. Akita and M. Yoshizawa, Angew. Chem., Int. Ed., 2013, 52, 8120-8123; (e) N. Kishi, Z. Li, Y. Sei, M. Akita, K. Yoza, J. S. Siegel and M. Yoshizawa, Chem.Eur. J., 2013, 19, 6313-6320; (f) N. Kishi, M. Akita, M. Kamiya, S. Hayashi, H.-F. Hsu and M. Yoshizawa, J. Am. Chem. Soc., 2013, 135, 12976-12979; ( $g$ ) N. Kishi, M. Akita and M. Yoshizawa, Angew. Chem., Int. Ed., 2014, 53, 36043607; (h) M. Yamashina, Y. Sei, M. Akita and M. Yoshizawa, Nat. Commun., 2014, 5, 4662; (i) K. Yazaki, Y. Sei, M. Akita and M. Yoshizawa, Nat. Commun., 2014, 5, 5179.

9 M. Yoshizawa and J. K. Klosterman, Chem. Soc. Rev., 2014, 43, 1885-1898. 
10 K. Hagiwara, Y. Sei, M. Akita and M. Yoshizawa, Chem. Commun., 2012, 48, 7678-7680.

11 M. F. Semmelhack, P. M. Helquist and L. D. Jones, J. Am. Chem. Soc., 1971, 93, 5908-5910.

12 Because of the existence of three atropisomers caused by hindered rotation of the two anthryl-phenyl bonds.

13 The proton NMR spectra of the host-guest complexes in $\mathrm{D}_{2} \mathrm{O}$ were also broadened and thus we confirmed the structures by detailed UV-vis and MS studies. Guests $\mathbf{4 a - d}$ are highly hydrophobic molecules and therefore we cannot determine the association constants under the heterogeneous conditions.

14 The $1 \supset(\mathbf{4 a})_{2}$ complex is stable enough under ESI-TOF MS conditions and therefore the spectrum showed a series of prominent peaks at e.g., $m / z=401.9\left[\mathbf{1} \supset(\mathbf{4 a})_{2}-7 \mathrm{Na}^{+}\right]^{7-}$, $472.7\left[\mathbf{1} \supset(\mathbf{4 a})_{2}-6 \mathrm{Na}^{+}\right]^{6-}$, and $571.7\left[\mathbf{1} \supset(\mathbf{4 a})_{2}-5 \mathrm{Na}^{+}\right]^{5-}$ (Fig. S19†).

15 Force-field calculations were carried out by the use of Materials Studio version 5.0 (Accelrys Software Inc., San Diego, CA).

16 (a) E. Arici, A. Greiner, H. Hou, A. Reuning and J. H. Wendorff, Macromol. Chem. Phys., 2000, 201, 20832090; (b) J.-A. Cheng, C.-P. Chang, C.-H. Chen and M.-S. Lin, J. Polym. Res., 2005, 12, 53-59; (c) W. Lin, L. Yuan and J. Feng, Eur. J. Org. Chem., 2008, 3821-3825.

17 The apparent FRETefficiencies of $\mathbf{1} \supset(\mathbf{4 a})_{2}$ and $\mathbf{1} \supset(\mathbf{4 b})_{2}$ were estimated to be $\sim 99 \%$ from the fluorescencequenching profile of the anthracene moieties (Fig. S23 and S29†). 